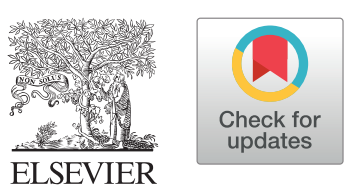

CJC Open 3 (2021) S9-S11

\title{
Editorial
}

\section{A Prevention Approach to Reducing Gender-Based Harassment and Discrimination in Cardiovascular Medicine}

\author{
Rachel Eikelboom, MD, and Sonia S. Anand, MD, FRCPC, $\mathrm{PhD}^{\mathrm{b}}$ \\ ${ }^{a}$ Department of Surgery, Max Rady College of Medicine, University of Manitoba, Winnipeg, Manitoba, Canada \\ ${ }^{b}$ Department of Medicine, McMaster University, Hamilton, Ontario, Canada
}

Gender harassment (physical or verbal behaviour, such as bullying) and discrimination (being treated differently on the basis of gender) are rampant in healthcare. Gender discrimination in the workplace has 4 key manifestations: creation of a hostile work environment, a gender pay gap, inequalities in career advancement, and imbalanced representation in leadership. Half of the women in healthcare training or academic healthcare witness or experience unprofessional behaviour. ${ }^{1}$ One in 2 female medical students (compared to 1 in 5 male medical students), ${ }^{2}$ and 1 in 3 female physicians (compared to $4 \%$ of male physicians) ${ }^{3}$ experience sexual harassment. Women in healthcare are less likely to be promoted than their male colleagues, despite having similar seniority and productivity, ${ }^{4}$ and women in academic medicine are paid less than men for the same job. ${ }^{5}$ Women continue to be underrepresented in upper faculty ranks, with no evident improvement since $1980 .^{\circ}$

Gender-based disparities in healthcare are particularly prominent in cardiovascular medicine. In Canada, where more than $50 \%$ of medical students are women, only $30 \%$ of trainees in cardiology and cardiovascular surgery residency programs are women. Only 22\% of cardiologists and $9 \%$ of cardiac surgeons are women, and there are no women who are heads of departments or residency program directors in cardiac surgery at present. Surveys of female trainees suggest that the underrepresentation of women in cardiovascular residency training programs is influenced by observations of a culture that favour male stereotypes and agentic personality types, and is not welcoming for women who plan to have children. ${ }^{8}$ Furthermore, the "leaky pipeline" ${ }^{9}$ phenomenon, a metaphor which describes the attrition of women from the academic pipeline before they reach senior positions, reflects

Received for publication June 15, 2021. Accepted September 8, 2021.

Ethics Statement: This research report has adhered to the relevant ethical guidelines.

Corresponding author: Dr Sonia S. Anand, Department of Medicine, McMaster University, 1280 Main Street West, Hamilton, Ontario L8S 4K1, Canada. Tel.: +1-905-525-9140.

E-mail: anands@mcmaster.ca

See page S11 for disclosure information. the inadequacy of current strategies to reduce gender harassment and discrimination.?

The most common strategies to address gender discrimination are professionalism policies, internal grievance systems, and implicit bias training. Clinicians are often unaware of the existence of professionalism policies, and those who are aware may not report unprofessional behaviour because they doubt their report will lead to action by hospitals or universities. Internal grievance systems, typically available through human resource departments, are underutilized because people are concerned that lack of anonymity will result in negative repercussions for reputation and career advancement. ${ }^{10}$ In turn, underutilization falsely reassures organizations that harassment and discrimination are not happening. Implicit bias training is a short-term educational intervention that may in fact worsen biases and discrimination by activating stereotypes. ${ }^{11}$ There is little evidence that implicit bias training brings about institutional change or reduces harassment and discrimination in the workplace. ${ }^{12}$ However, organizations have unrealistic confidence in implicit bias training programs, which desensitizes them to persistent or even worsening biases. ${ }^{13}$

When professionalism policies, internal grievance systems, and implicit bias training fail, cases of gender harassment and discrimination result in formal complaints to administrators, regulatory bodies, professional societies, and human rights tribunals. ${ }^{14}$ By this time, harassment and discrimination are so advanced that all possible outcomes result in damage to both complainant and institutions, as well as significant collateral damage such as impact on staff and faculty morale. Here, as in the prevention of cardiovascular disease, we believe "an ounce of prevention is worth a pound of cure." Primary prevention of cardiovascular disease is effective, especially in high-risk patients with a strong family history of premature cardiovascular disease or those with risk factors. Analogously, primary prevention strategies should be initiated in high-risk healthcare environments, in which bullying, cronyism, and severe imbalances of gender should be considered risk factors.

Primary prevention of gender harassment and discrimination should focus on the creation of a more diverse and inclusive work environment. Increased representation of women, and diverse gender, ethnicity, and race identities, in the workplace promotes an institutional climate less tolerant of 


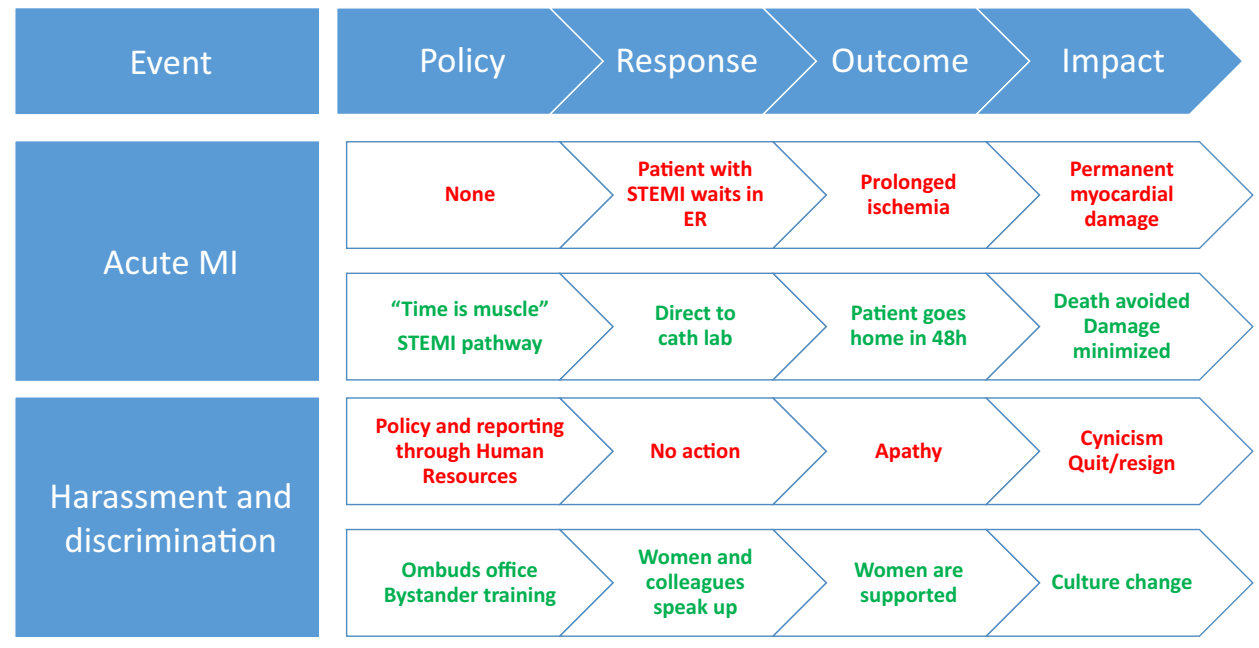

Figure 1. Cardiovascular and discrimination pathways and outcomes. ER, emergency room; MI, myocardial infarction; STEMI, ST-segment elevation myocardial infarction.

traditional decision-making networks such as the "old boys' club." Diversity in the workplace also prevents "group-think" and blind spots, so that issues such as the gender pay gap and unequal career advancement are identified and discussed. ${ }^{15}$ Structural changes to create more-inclusive work environments include stronger top-down modelling, discussion of professionalism, and the creation of equitable practices for leadership opportunities and remuneration. A diverse and inclusive work environment is also promoted by informal actions such as the "cup of coffee approach" between the leadership and suggested perpetrators of harassment or discrimination, in order to convey that such behaviour is noticed and is unacceptable.

However, as with the prevention of cardiovascular disease, primary prevention strategies for gender harassment and discrimination are often imperfect, and secondary prevention strategies are required to address persistent symptoms and manifestations. Akin to coordinated ST-segment elevation myocardial infarction (STEMI) pathways, which by reducing ischemic, time minimize myocardial damage and dysfunction, effective secondary prevention pathways to address gender harassment and discrimination are not commonly available in hospital systems and academic medical institutions in Canada. Unreliable secondary prevention pathways, such as professionalism policies that are not enforced and internal grievance systems that cannot maintain confidentiality, instead can result in more damage and disease progression (Fig. 1).

One effective secondary prevention strategy is bystander training, which has been successfully implemented on American college campuses and in the military. ${ }^{11}$ Bystander training creates a culture of accountability, where everyone in the workplace assumes responsibility for speaking up against harassment. Bystander training removes the onus from those experiencing harassment and discrimination, and spreads responsibility, agency, and engagement across all members of the workplace.

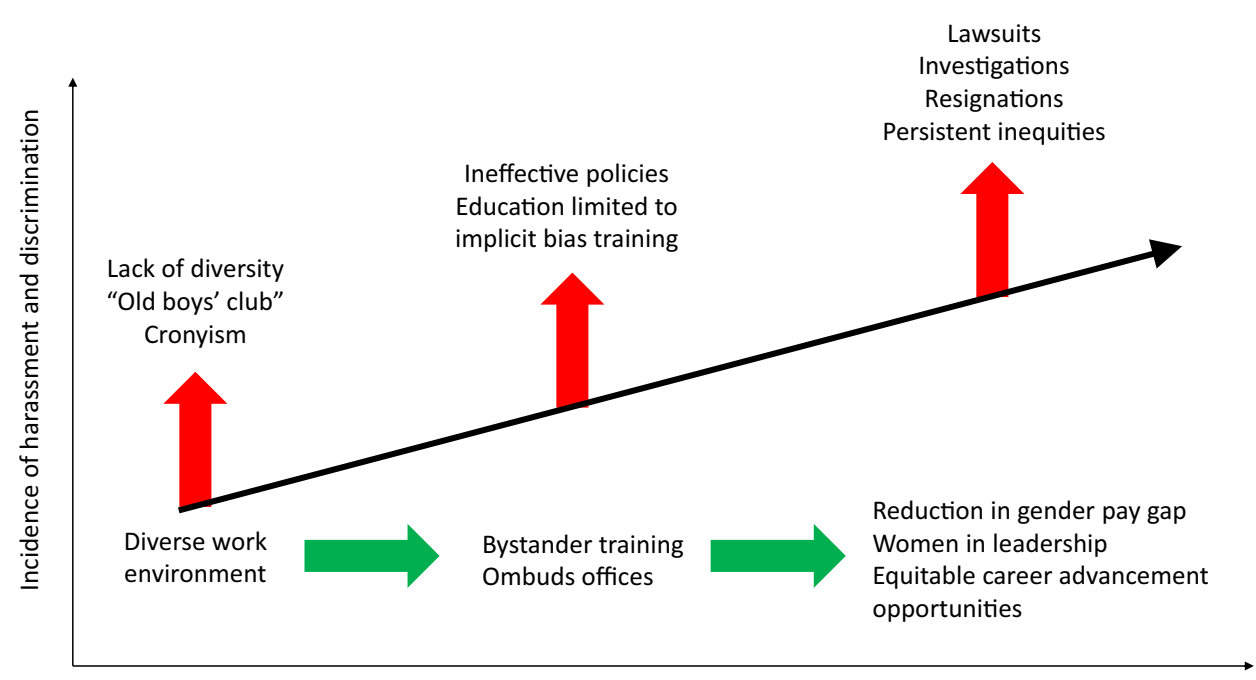

Figure 2. Pathway by which a more gender-balanced and equitable workplace environment can reduce the gender pay gap and increase career advancement and leadership opportunities for women in cardiovascular medicine and surgery. 
Those experiencing harassment and discrimination also require a safe and confidential way to speak up. Ombuds offices are independent, confidential, neutral, and informal resources for organizations and their employees. They hear employees' concerns confidentially and help them to think through their options. They also compile anonymous information on hotspots, trends, and systemic issues for organizations to target. ${ }^{16}$ Setting up an ombuds office is not as difficult or costly as administrators may think: such offices are even available online through virtual ombuds services, such as tEQuitable, a Silicon Valley startup. ${ }^{17}$

Primary and secondary prevention of gender harassment and discrimination in medicine requires innovative strategies that create cultural and structural change. As with strategies to prevent cardiovascular disease, these prevention strategies should be evidence-based, and their impact must be measured and reported. Only by changing their culture and structure will cardiovascular medicine and surgery departments see significant improvements in the workplace environment for women. Developing and evaluating clear strategies to reduce harassment and discrimination (ie, bystander training and ombuds offices) will shift culture and create a work environment that does not dissuade female trainees from pursuing cardiovascular medicine and surgery. The creation of a more gender-balanced and equitable workplace environment should lead to a reduction in the gender pay gap, and increase career advancement and leadership opportunities for women in cardiovascular medicine and surgery (Fig. 2).

\section{Funding Sources}

The authors have no funding sources to declare.

\section{Disclosures}

The authors have no conflicts of interest to disclose.

\section{References}

1. Leape LL, Shore MF, Dienstag JL, et al. Perspective: a culture of respect, part 2: creating a culture of respect. Acad Med 2012;87:853-8.

2. Dzau VJ, Johnson PA. Ending sexual harassment in academic medicine. N Engl J Med 2018;379:1589-91.
3. Jagsi R, Griffith KA, Jones R, et al. Sexual harassment and discrimination experiences of academic medical faculty. JAMA 2016;315:2120-1.

4. Chisholm-Burns MA, Spivey CA, Hagemann T, Josephson MA. Women in leadership and the bewildering glass ceiling. Am J Health Syst Pharm 2017;74:312-24.

5. Jena AB, Olenski AR, Blumenthal DM. Sex differences in physician salary in US public medical schools. JAMA Intern Med 2016;176: 1294-304.

6. Richter KP, Clark L, Wick JA, et al. Women physicians and promotion in academic medicine. N Engl J Med 2020;383:2148-57.

7. Banks L, Randhawa VK, Caterini J, et al. Sex, gender, and equity in cardiovascular medicine, surgery, and science in Canada: challenges, successes, and opportunities for change. CJC Open 2020;2:522-9.

8. Douglas PS, Rzeszut AK, Bairey Merz CN, et al. Career preferences and perceptions of cardiology among US internal medicine trainees: factors influencing cardiology career choice. JAMA Cardiol 2018;3:682-91.

9. Goulden M, Mason MA, Frasch K. Keeping women in the science pipeline. Ann Am Acad Polit Soc Sci 2011;638:141-62.

10. Wear D, Aultman J. Sexual harassment in academic medicine: persistence, non-reporting, and institutional response. Med Educ Online 2005;10:4377.

11. Dobbin F, Kalev A. Why diversity training doesn't work: the challenge for industry and academia. Anthropol Now 2018;10:48-55.

12. Bezrukova K, Spell CS, Perry JL, Jehn KA. A meta-analytical integration of over 40 years of research on diversity training evaluation. Psychol Bull 2016;142:1227-74.

13. Brady LM, Kaiser CR, Major B, Kirby TA. It's fair for us: Diversity structures cause women to legitimize discrimination. J Exp Soc Psychol 2015;57:100-10.

14. Choo EK, van Dis J, Kass D. Time's up for medicine? Only time will tell. N Engl J Med 2018;379:1592-3.

15. Holvino E, Ferdman BM, Merrill-Sands D. Creating and sustaining diversity and inclusion in organisations: strategies and approaches. In: Crosby MSSFJ, ed. The Psychology and Management of Workplace Diversity. Malden, MA: Blackwell Publishing, 2004:245-76.

16. Howard C. The Organizational Ombudsman: Origins, Roles, and Operations. A Legal Guide. Chicago, IL: American Bar Association, 2009.

17. tEQuitable. tEQuitable website. Available at: https://www.tequitable. com. Accessed June 3, 2021. 